\title{
A systematic review of hereditary spherocytosis reported in Chinese biomedical journals from 1978 to 2013 and estimation of the prevalence of the disease using a disease model
}

\author{
Chao Wang ${ }^{1,2}$, Yazhou Cui ${ }^{1}$, Yan $\mathrm{Li}^{1,2}$, Xiao Liu ${ }^{1,2}$, Jinxiang Han ${ }^{1, *}$ \\ ${ }^{1}$ Key Laboratory for Biotech Drugs of the Ministry of Health, Key Laboratory for Rare Disease of Shandong Province, Shandong \\ Medicinal Biotechnology Center, Shandong Academy of Medical Sciences, Ji'nan, Shandong, China; \\ ${ }^{2}$ School of Medicine and Life Sciences, University of Jinan-Shandong Academy of Medical Science, Ji'nan, Shandong, China.
}

\begin{abstract}
Summary Epidemiological information of hereditary spherocytosis in China is slight. This systematic review summarizes the number of cases of hereditary spherocytosis reported in China Biology Medicine disc from 1978 to 2013. In total, 2,043 cases were reported in the past 36 years. We describe its distribution from time and space. We also estimate the literature reported prevalence of hereditary spherocytosis by DisMod-II software, overall prevalence in China was estimated to be: 1.27 cases per 100,000 people in males and 1.49 cases per $\mathbf{1 0 0 , 0 0 0}$ people in females. All results suggest a stronger network of diagnosis and treatment including all levels of hospitals should be created to improve healthcare for hereditary spherocytosis and even other rare diseases in the future, meanwhile we can obtain more useful information for orphan drug designation purposes and make public health decisions regarding such diseases through the use of the burden of disease models.
\end{abstract}

Keywords: Hereditary spherocytosis, Bibliographic study, DisMod-II, China

\section{Introduction}

Hereditary spherocytosis refers to a group of heterogeneous inherited anemias that are characterized by anemia, jaundice, and splenomegaly. The clinical situation of the disease is often most severe during the first year of life, and most newborns have severe anemia, although it is improved after the first year of life. Jaundice is the first clinical situation in newborns, with severe anemia developing during the first few days after birth. Splenomegaly is a frequently observed feature (1). The age of onset and severity of the disease vary considerably. Splenectomy is the first choice of treatment in some patients with symptomatic anemia as it eliminates the necessity of repeated transfusions. Recent

Released online in J-STAGE as advance publication March $31,2015$.

*Address correspondence to:

Dr. Jinxiang Han, Key Laboratory for Rare Disease Research of Shandong Province, Key Laboratory for Biotech Drugs of the Ministry of Health, Shandong Medical Biotechnological Center, Shandong Academy of Medical Sciences, Ji'nan, Shandong 250062, China.

E-mail: samshjx@sina.com evidence is emerging that splenectomy may have adverse vascular long term consequences, and whether patients should undergo splenectomy is controversial $(2,3)$.

Hereditary spherocytosis is reported worldwide and is the most common hereditary anemia in individuals from northern Europe and northern America (4-10). The prevalence of the disease is around 1 in 5,000 to 1 in 2,000 in the above two areas. The prevalence of hereditary spherocytosis in people of other ethnic backgrounds is unknown, but it is much less common. As a kind of rare disease, there is no exact prevalence of hereditary spherocytosis in China. Although China is also actively promoting regulation of rare diseases, the diseases have not been covered by the national health system. However, there are still no official data on "the prevalence of hereditary spherocytosis and the number of cases". So a crucial step is to collect data on hereditary spherocytosis in China. We searched the reported literature or cases in hospitals to obtain the data collection from China.

This study systematically reviewed hereditary spherocytosis reported in the Chinese biomedical literature published over the past 36 years from January 1978 to December 2013 and analyzed the current state and treatment of hereditary spherocytosis in China. 
This study also estimated the rough literature reported prevalence of hereditary spherocytosis in China.

\section{Methods}

\subsection{Data collection}

\subsubsection{The literature data}

We searched the literature related to hereditary spherocytosis from China Biology Medicine disc (CBMdisc) (http://www.sinomed.ac.cn/) that covered articles since January 1978 to December 2013. The CBM database is the largest Chinese biomedical bibliographic database, it includes more than 1,600 kinds of biomedical journals, conference papers and compilations published in Chinese, and includes 7,875,309 literature citations prior to 2013 .

Chinese terms and English terms describing hereditary spherocytosis were used to search for publications in the CBM database (11). English terms for hereditary spherocytosis were included because most Chinese biomedical publications contain an English abstract. We collected literature related to clinical data, diagnostic information and laboratory data searched in the CBM database. Cases of hereditary spherocytosis with a confirmed diagnosis were included. For each study included, informed consent for publication was obtained from the patient. Patient medical information was carefully compared for series of reports by the same authors or institutions, and redundant cases were excluded.

\subsubsection{Other data}

The World Health Organization (WHO) and Harvard University designed DisMod-II software to calculate the burden of diseases. The software was used to estimate the prevalence of a simple disease model with four possible kinds of data, namely: incidence; mortality of the disease; all other mortality and remission. The model permits calculation of prevalence at a certain age of remission, the mortality and incidence in the age interval (12).

The mortality of hereditary spherocytosis was selected from CDC WONDER (Centers for Disease Control and Prevention Wide-Ranging Online Data for Epidemiologic Research) (http://wonder.cdc.gov/). The CDC WONDER is the nation's primary data repository for health statistics of the United States, which covers the CDC site data and statistical information service. Through the system, users can retrieve the morbidity and public health data including, scientific research data, survey data, health statistical data, laboratory information and so on (13).

All other mortality was collected from the national disease surveillance system death surveillance data sets
2011 which was written by Chinese Center for Disease Control and Prevention that summarizes the national disease surveillance systems (DSPs) 2011 round of population and mortality data (14).

The structure of the general population of China was obtained by the sixth national population census of the People's Republic of China, which was published in the National Bureau of Statistics of the People's Republic of China in 2010 (15). It is an agency within the State Council of the People's Republic of China charged with the collection and publication of statistics related to the economy, population and society of the People's Republic of China at national and local levels.

\subsection{Statistical analysis}

\subsubsection{The literature data}

SPSS 21.0 was used to input and manage data, it was corrected in accordance with the original data when there was abnormal data or missing values. Descriptive statistical analysis was used for the frequency distribution analysis and description of the data characteristics. Charts were drawn with SPSS and Excel. A map with shades of color reflecting the number of hereditary spherocytosis cases in each city was created with ArcMap 10.0.

\subsubsection{Prevalence of hereditary spherocytosis}

We selected year 2011 for calculation purposes as this was the latest year for which all other mortality data of China were available. The patients of hereditary spherocytosis were selected from the literature data in 2011, then we used the number of patients from 2011 and the structure of the general population of China to calculate incidence. All data were classified by gender and age group because of the requirements of DisModII software.

The Multiple Cause of Death database contains mortality and population counts for all USA counties from 1999 to 2013 . We extracted deaths from hereditary spherocytosis of 15 years data, then calculated the average mortality which were broken down by gender and age group.

Hereditary spherocytosis is the only congenital hemolytic anemia for which a splenectomy proves consistently beneficial. Recent evidence is emerging that there may be adverse long term vascular consequences of splenectomy, whether patients should undergo splenectomy is controversial and splenectomy does not eradicate birth defects. So remission was assumed to be zero.

Finally, the model was constructed using hereditary spherocytosis mortality data in USA, and remission. All other mortality was also introduced into the model, along with the national disease surveillance system 
Table 1. Estimated prevalence and incidence of hereditary spherocytosis by age group, based on incidence in Chinese literature reported and mortality from USA

\begin{tabular}{|c|c|c|c|c|c|c|c|c|}
\hline \multirow{3}{*}{ Age group } & \multicolumn{4}{|c|}{$\operatorname{Input}(\times 100,000)^{*}$} & \multicolumn{4}{|c|}{ Output $(\times 100,000)$} \\
\hline & \multicolumn{2}{|c|}{ Mortality\& $^{\&}$} & \multicolumn{2}{|c|}{ Incidence } & \multicolumn{2}{|c|}{ Prevalence } & \multicolumn{2}{|c|}{ Incidence } \\
\hline & Males & Females & Males & Females & Males & Females & Males & Females \\
\hline$<1$ & 0.0131 & 0.0069 & 0.0804 & 0.1423 & 0.0179 & 0.0188 & 0.0359 & 0.0377 \\
\hline $1-4$ & 0.0000 & 0.0000 & 0.0298 & 0.0320 & 0.1089 & 0.1141 & 0.0377 & 0.0390 \\
\hline $5-14$ & 0.0006 & 0.0003 & 0.0457 & 0.0432 & 0.3900 & 0.3947 & 0.0364 & 0.0354 \\
\hline $15-24$ & 0.0003 & 0.0010 & 0.0026 & 0.0027 & 0.5715 & 0.5697 & 0.0052 & 0.0049 \\
\hline $25-34$ & 0.0010 & 0.0003 & 0.0000 & 0.0020 & 0.6121 & 0.5964 & 0.0045 & 0.0025 \\
\hline $35-44$ & 0.0006 & 0.0003 & 0.0016 & 0.0008 & 0.6387 & 0.6117 & 0.0024 & 0.0015 \\
\hline $45-54$ & 0.0019 & 0.0012 & 0.0011 & 0.0033 & 0.4583 & 0.6279 & 0.0014 & 0.0037 \\
\hline $55-64$ & 0.0004 & 0.0020 & 0.0000 & 0.0000 & 0.3646 & 1.2525 & 0.3828 & 0.2031 \\
\hline $65-74$ & 0.0000 & 0.0031 & 0.0000 & 0.0000 & 3.1813 & 5.5225 & 0.3501 & 0.7011 \\
\hline $75-84$ & 0.0063 & 0.0052 & 0.0000 & 0.0000 & 11.0010 & 14.3372 & 2.5060 & 0.8380 \\
\hline $85+$ & 0.0343 & 0.0059 & 0.0000 & 0.0000 & 86.3382 & 22.7439 & 4.8329 & 0.6042 \\
\hline Total & 0.0011 & 0.0011 & NA & NA & 1.2669 & 1.4862 & 0.1508 & 0.0993 \\
\hline
\end{tabular}

${ }^{*}$ Remission input (assumed to be zero). ${ }^{\&}$ Mortality in USA.

death surveillance data sets 2011 of China. Then the ensuing estimates of prevalence were shown by age group and gender.

\section{Results}

373 reports were searched in CBM database, 297 reports were qualified for inclusion according to our criteria. A total of 2,043 cases of hereditary spherocytosis were reported in the literature. As shown in Figure 1, the number of patients with hereditary spherocytosis reported each year in the CBM database, after 1994 is significantly higher than a few years ago. $71 \%$ of the cases were diagnosed at a university hospital, $22 \%$ were diagnosed at a municipal hospital, $4 \%$ were diagnosed at a provincial hospital, and the remainder $(3 \%)$ was diagnosed at hospitals on country level or even from smaller communities (Figure 2). The geographic distribution of reported patients is shown in Figure 3. From the map view, more cases were reported in the South and East of China, which have a higher population density and better medical services than other areas. Shandong, Beijing, Liaoning, Hebei and Shanghai ranked among the top 5 provinces or province level municipalities where disorders were reported. In Xizang, Qinghai and Guizhou only a few cases were reported.

In 2011, the number of cases was 114 reported from CBM database, the male: female ratio was 1.04:1. According to the sixth national population census of the people's republic of China, Chinese population totaled $1,332,810,869$, with a breakdown by sex of $682,329,104$ males and 650,481,765 females. In 2011, overall literature reported prevalence of hereditary spherocytosis in China was estimated to be: 1.27 cases per 100,000 people in males and 1.49 cases per 100,000 people in females (Table 1). From the table we found that the model estimated incidence of hereditary spherocytosis was high before the age of 15 and after

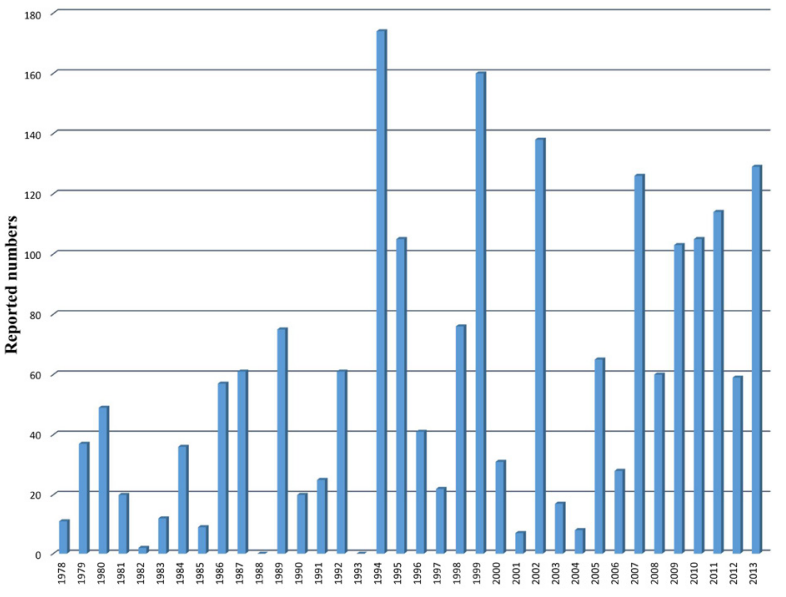

Figure 1. Case numbers of hereditary spherocytosis reported in Chinese biomedical publications from 1978 to 2013. The number of hereditary spherocytosis cases has increased significantly after 1994, and in the last 10 years there are 979 cases that accounted for nearly $1 / 2$ of the reported numbers.

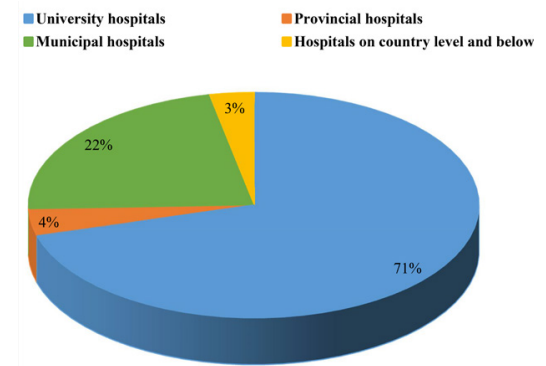

Figure 2. Hospital distribution of reported cases of hereditary spherocytosis in Chinese biomedical Publications. $71 \%$ of the cases were diagnosed at a university hospital, $22 \%$ at a municipal hospital, $4 \%$ at a provincial hospital, and the remainder $(3 \%)$ was diagnosed at hospitals on country level or even from smaller communities.

the age of 55. We also estimated that China has 8,644 males and 9,667 females hereditary spherocytosis sufferers (Figure 4). The prevalence calculated from national and literature data by the DisMod-II software 


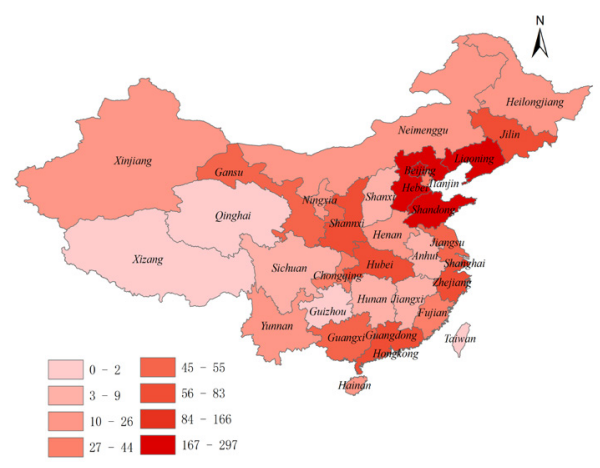

Figure 3. Geographic distribution of reported cases of hereditary spherocytosis in Chinese biomedical publications. More cases were reported in the South and East of China, Shandong, Beijing, Liaoning, Hebei and Shanghai ranked among the top 5 provinces or province level municipalities where cases were reported. Xizang, Qinghai and Guizhou only a few cases were reported.

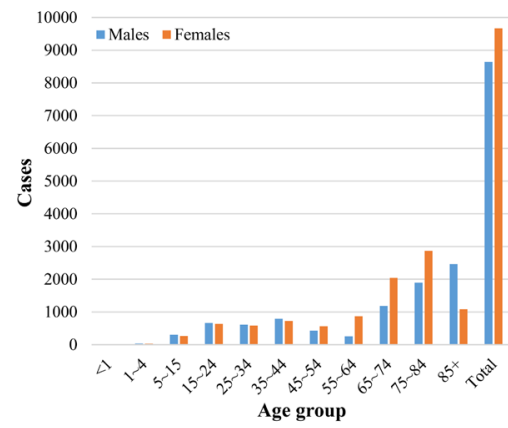

Figure 4. Estimation of hereditary spherocytosis prevalent cases by gender and age groups in China. China was estimated to have 8,644 males and 9,667 females of hereditary spherocytosis sufferers according to the number of hereditary spherocytosis cases reported in Chinese biomedical publications.

is shown in Figure 5. The model estimates an increase with age but with a slower pace, after 65 years cases increase suddenly and at ages $>85$ years the estimates reach a maximum.

\section{Discussion}

Hereditary spherocytosis is a rare disease, there is no cure for hereditary spherocytosis caused by genetic defect, and thus the focus of current management is to limit the severity of the disease. Treatment options include: splenectomy and partial splenectomy, even cholecystectomy. Splenectomy is the most effective way of treatment and will be of benefit in all people with severe as well as some people with moderate hereditary spherocytosis, but is not usually necessary in mild cases. Recent evidence demonstrates that splenectomy for hereditary spherocytosis is safe in the short term, studies showed no deaths and infrequent complications in 1,657 children (16). The disadvantages of splenectomy are large trauma and significant immunity decline. Partial splenectomy applied to the young, yields low immunity, and splenectomy might cause serious infection of patients who may relapse

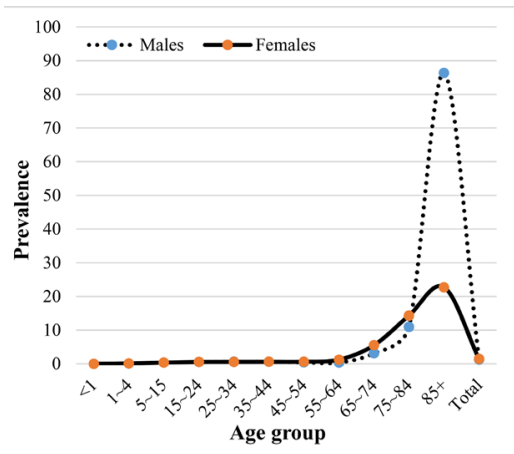

Figure 5. Estimated prevalence of hereditary spherocytosis by gender and age groups in China. Model estimates increase with age but at a slower pace, after 65 years increases suddenly and at ages $>85$ years the estimates reach a maximum.

again. Investigators finished a follow-up of 10 years and found that $7.5 \%$ cases needed a total splenectomy again (17).

From the results of publications in CBM database, the number of hereditary spherocytosis cases increased significantly after 1994 , and in the last 10 years there were 979 cases that accounted for nearly $1 / 2$ of the reported numbers. This situation is because general healthcare has improved rapidly and people pay more attention to medical problems caused by rare diseases in China. Cases were more frequently reported around the Bohai area which contains Beijing, Shandong, Liaoning and Hebei. We noted that more university hospitals and blood institutes are in these provinces. Also cases were more reported in large cities instead of areas with a larger population but a relatively lower level of development such as Guizhou and Sichuan Provinces and even Xizang found no reported cases. Country level hospitals and below represent more than $70 \%$ of the medical resources in China, but only $3 \%$ of the cases of hereditary spherocytosis were diagnosed by these hospitals. There are also some disparities between university hospitals and provincial and municipal hospitals. So, countries should pay more attention to reform health care and improve the level of medical technology of country level hospitals and below. At the same time, a stronger network of diagnosis and treatment including all levels of hospitals should be created to improve healthcare for hereditary spherocytosis and other rare diseases in the future.

Although hereditary spherocytosis is encountered worldwide, its prevalence in other groups (excluding northern European and northern American groups) has not been clearly established. Currently, there is no case registration system for most rare diseases in China, so there is very little information on the epidemiology of hereditary spherocytosis. To our knowledge, a systematic survey of the literature was being performed in order to provide an estimated prevalence of rare diseases or the reported number of published cases in Europe. The prevalence of hereditary spherocytosis was $1 / 5,000$ in Europe which was published on orphanet (the portal for 
rare diseases and orphan drugs) in May 2014 (18).

DisMod-II was designed as a system to assist in the burden of disease models in the 1990s, and in a few exceptional cases, to estimate disease prevalence (19-21). DisMod-II has seven kinds of possible input variables: incidence, remission, case fatality rate, mortality, prevalence, duration and relative risk of death. In general, three input variables are needed to calculate the other four variables and the age of onset. When there are less than three available variables, we can use expert judgment to obtain additional information. The greatest source of error may be from using information that has a lack of data or a small sample size that represents large groups. But the model is fairly robust, it will reasonably allow an estimate of the prevalence, which is comparable with figures reported by published studies. Traditional prevalence studies require more time to collect and calculate data, and only allow input of the number of cases and time or incidence and duration. Nevertheless, DisMod-II permits modelling of variability situations, such as entering different models of remission, incidence and case fatality rate. DisMod-II should be the most effective way to estimate the prevalence of rare diseases.

The model is based on the assumption that incidence and mortality are in a steady state, but it is dependent on the incidence and mortality from the past as well as the present (22). The incidence of hereditary spherocytosis was collected and calculated from CBM database reported in 2011, although it would not represent all cases. The mortality of hereditary spherocytosis was selected from CDC WONDER and was an average mortality between 1999 and 2013 in USA. In conclusion, our analysis suggests that previous estimates of prevalence, based on smaller datasets, have underestimated the prevalence of hereditary spherocytosis in China. In the future, we can collect more information from medical record registration systems in hospitals to estimate an accurate prevalence.

\section{Conclusion}

In conclusion, this systematic review summarized the number of cases and geographic areas of hereditary spherocytosis in China Biology Medicine disc and also estimated the literature reported prevalence of hereditary spherocytosis by DisMod-II software in China. Analyzing the number of cases indicated an imbalance in the distribution of areas and hospitals diagnosis, which suggests a stronger network of diagnosis and treatment including all levels of hospitals that should be created to improve healthcare for hereditary spherocytosis and even other rare diseases in the future. Then we can have more accurate information to estimate prevalence. In addition, through DisModII software we can obtain more useful information for orphan drug designation purposes and make public health decisions regarding such diseases.

\section{Acknowledgements}

This study was supported by Key Projects in the National Science \& Technology Pillar Program during the Twelfth Five-year Plan Period (2013BAI07B01).

\section{References}

1. Perrotta S, Gallagher PG, Mohandas N. Hereditary spherocytosis. Lancet. 2008; 372:1411-1426.

2. Shah A. Hemoglobinopathies and other congenital hemolytic anemia. Indian J Med Sci. 2004; 58:490-493.

3. Bolton-Maggs PH, Langer JC, Iolascon A, Tittensor P, King MJ; General Haematology Task Force of the British Committee for Standards in Haematology. Guidelines for the diagnosis and management of hereditary spherocytosis--2011 update. Br J Haematol. 2012; 156:3749.

4. Bogusławska DM, Heger E, Chorzalska A, Nierzwicka M, Hołojda J, Swiderska A, Straburzyńska A, Paździor G, Langner M, Sikorski AF. Hereditary spherocytosis: Identification of several HS families with ankyrin and band 3 deficiency in a population of southwestern Poland. Ann Hematol. 2004; 83:28-33.

5. Godal HC, Heistø H. High prevalence of increased osmotic fragility of red blood cells among Norwegian blood donors. Scand J Haematol. 1981; 27:30-34.

6. Jensson O, Jónasson JL, Magnússon S. Studies on hereditary spherocytosis in Iceland. Acta Med Scand. 1977; 201:187-195.

7. Eber SW, Pekrun A, Neufeldt A, Schröter W. Prevalence of increased osmotic fragility of erythrocytes in German blood donors: Screening using a modified glycerol lysis test. Ann Hematol. 1992; 64:88-92.

8. Premetis E, Stamoulakatou A, Loukopoulos D. Erythropoiesis: Hereditary spherocytosis in Greece: Collective data on a large number of patients. Hematology. 1999; 4:361-366.

9. Sánchez-López JY, Camacho AL, Magaña MT, Ibarra B, Perea FJ. Red cell membrane protein deficiencies in Mexican patients with hereditary spherocytosis. Blood Cells Mol Dis. 2003; 31:357-359.

10. Ricard MP, Gilsanz F, Millan I. Erythroid membrane protein defects in hereditary spherocytosis. A study of 62 Spanish cases. Haematologica. 2000; 85:994-995.

11. Cui Y, Zhao H, Liu Z, Liu C, Luan J, Zhou X, Han J. A systematic review of genetic skeletal disorders reported in Chinese biomedical journals between 1978 and 2012. Orphanet J Rare Dis. 2012; 7:55.

12. Kruijshaar ME, Barendregt JJ, Hoeymans N. The use of models in the estimation of disease epidemiology. Bull World Health Organ. 2002; 80:622-628.

13. Friede A, Reid JA, Ory HW. CDC WONDER: A comprehensive on-line public health information system of the Centers for Disease Control and Prevention. Am J Public Health. 1993; 83:1289-1294.

14. Chinese Center for Disease Control and Prevention. The national disease detection systems death surveillance data set (Yu Wang, Fu Gao, eds.). People's Medical Publishing House, BJ, China, 2011.

15. National Bureau of Statistics of the People's Republic of China (2010). http://www.stats.gov.cn/tjsj/pcsj/rkpc/6rp/ indexch.htm (accessed February 1, 2015). (in Chinese) 
16. Abdullah F, Zhang Y, Camp M, Rossberg MI, Bathurst MA, Colombani PM, Casella JF, Nabaweesi R, Chang DC. Splenectomy in Hereditary Spherocytosis: Review of 1,657 Patients and Application of the Pediatric Quality Indicators. Pediatr Blood Cancer. 2009; 52:834-837.

17. Bader-Meunier B, Gauthier F, Archambaud F, Cynober T, Miélot F, Dommergues JP, Warszawski J, Mohandas N, Tchernia G. Long-term evaluation of the beneficial effect of subtotal splenectomy for management of hereditary spherocytosis. Blood. 2001; 97:399-403.

18. ORPHANET. Prevalence of rare diseases: Bibliographic data. http://www.orpha.net/orphacom/cahiers/docs/ GB/Prevalence_of_rare_diseases_by_alphabetical_list (accessed February 1, 2015). (in Chinese).

19. Villaverde-Hueso A, de la Paz MP, Martín-Arribas MC, Sánchez-Valle E, Ramírez-González A, Biairdi P.
Prevalence of scleroderma in Spain: An approach for estimating rare disease prevalence using a disease model. Pharmacoepidemiol Drug Saf. 2008; 17:1100-1107.

20. Kruijshaar ME, Barendregt JJ, Van De Poll-Franse LV. Estimating the prevalence of breast cancer using a disease model: Data problems and trends. Popul Health Metr. 2003; 1:5.

21. Wild S, Roglic G, Green A, Sicree R, King H. Global prevalence of diabetes: Estimates for the year 2000 and projections for 2030. Diabetes Care. 2004; 27:1047-1053.

22. Murray CJ, Lopez AD. Quantifying disability: Data, methods and results. Bull World Health Organ. 1994; 72:481-494.

(Received February 5, 2015; Revised March 10, 2015; Accepted March 18, 2015) 